BULL. AUSTRAL. MATH. SOC.

VOL. 19 (1978), 457-466.

\title{
Linear approximation by primes
}

\section{Kee-Wai Lau and Ming-Chit Liu}

In this present paper we shall prove the following. Suppose that $\lambda_{1}, \lambda_{2}, \lambda_{3}$ are any non-zero real numbers not all of the same sign and that $\lambda_{1} / \lambda_{2}$ is irrational. If $\eta$ is any real number and $0<\alpha<1 / 9$, then there are infinitely many prime triples $\left(p_{1}, p_{2}, p_{3}\right)$ for which

$$
\left|n+\lambda_{1} p_{1}+\lambda_{2} p_{2}+\lambda_{3} p_{3}\right|<\left(\max p_{j}\right)^{-\alpha}
$$

\section{Introduction}

Suppose that $\lambda_{1}, \ldots, \lambda_{s}$ are any non-zero real numbers, not all of the same sign and not all in rational ratio. In 1946, Davenport and Heilbronn [3] proved that if $k$ is a positive integer and $s \geq 2^{k}+1$, then for any $\varepsilon>0$ the inequality

$$
\left|\sum_{j=1}^{s} \lambda_{j} n_{j}^{k}\right|<\varepsilon
$$

has infinitely many solutions in integers $n_{j} \geq 1$. This result sparked off a series of investigations (for information, see the introductions in [9], [10], [11]). Schwarz [8] was able to replace all the $n_{j}$ in (1.1) by primes $p_{j}$ and obtained a better lower bound for $s$ if $k \geq 12$. For the special case $k=1$, Baker [1] introduced a new kind of approximation by showing that for any number $A>0$ the inequality

$$
\left|\lambda_{1} p_{1}+\lambda_{2} p_{2}+\lambda_{3} p_{3}\right|<\left(\log \max p_{j}\right)^{-A}
$$

Received 17 October 1978. 
has infinitely many solutions in primes $p_{j}$. Later, Ramachandra [7] refined matters still more and replaced the $\left(\log \max p_{j}\right)^{-A}$ in (1.2) by $\exp \left(-\left(\log p_{1} p_{2} p_{3}\right)^{\frac{1}{2}}\right)$. Recently Vaughan [9, p.374] made remarkable progress by proving that the right hand side of (1.2) can be replaced by $\left(\max p_{j}\right)^{-1 / 10}\left(\log \max p_{j}\right)^{20}$. He also remarked that it is interesting that one can save as much as $1 / 10$ and on the generalized Riemann hypothesis, only $1 / 5$ may be saved. The object of this paper is to show that we can save as much as $1 / 9-\delta$ for any $\delta>0$. We have:

THEOREM. Suppose that $\lambda_{1}, \lambda_{2}, \lambda_{3}$ are any non-zero real numbers not al2 of the same sign and that $\lambda_{1} / \lambda_{2}$ is irrational. If $n$ is any real number and $0<\alpha<1 / 9$, then there are infinitely many prime triples $\left(p_{1}, p_{2}, p_{3}\right)$ for which

$$
\left|n+\lambda_{1} p_{1}+\lambda_{2} p_{2}+\lambda_{3} p_{3}\right|<\left(\max p_{j}\right)^{-\alpha}
$$

Our proof is a refinement of the elegant argument of Vaughan's [9] which, in general principle, is based on the method of Davenport and Heilbronn [3].

\section{Notation and definitions}

Throughout, $x$ is a real variable and $n, p$, with or without suffices, denote any positive integer and any prime respectively. Since $\lambda_{1} / \lambda_{2}$ is irrational it is known [4, Theorem 183] that there are infinitely many convergents $a / q$ with $(\alpha, q)=1,1 \leq q$, such that

$$
\left|\left(\lambda_{1} / \lambda_{2}\right)-(a / q)\right|<1 / 2 q^{2} \text {. }
$$

For the given $\alpha<1 / 9$, let $A$ be any constant with

$$
\max (1 / 5,2 \alpha)<A<2 / 9 \text {. }
$$

Put

$$
X=q^{1 /(1-2 A)}, \quad L=\log X,
$$

$$
\varepsilon=X^{-A / 2} L^{20},
$$


$(2.5)$

$$
\begin{aligned}
K_{\varepsilon} & = \begin{cases}\varepsilon^{2} & \text { if } x=0, \\
(\sin \pi \varepsilon x)^{2} /(\pi x)^{2} & \text { otherwise, }\end{cases} \\
e(x) & =\exp (i 2 \pi x),
\end{aligned}
$$

$$
S(x)=\sum_{p \leq X} e(p x) \log p, S_{j}(x)=S\left(\lambda_{j} x\right),
$$

$$
F(x)=\prod_{j=1}^{3} S_{j}(x)
$$

Throughout, $\delta>0$ is a small number, and constants implied by the symbols $\ll$ and $\gg$ may depend on $\lambda_{j}, \delta, \eta$, and $A$ only.

Let $\tau_{1}=X^{A-1}, \tau_{2}=\delta X^{1-4 A}, \tau_{3}=X^{A}$, and $E_{1}=\left\{x:|x| \leq \tau_{1}\right\}$, $E_{2}=\left\{x: \tau_{1}<|x| \leq \tau_{2}\right\}, E_{3}=\left\{x: \tau_{2}<|x| \leq \tau_{3}\right\}$, $E_{4}=\left\{x: \tau_{3}<|x|\right\}$.

Here we partition the real line into four regions instead of the usual three regions such as in [9]. Our new region, $E_{3}$, is similar to the range (20) in [2]. By introducing such a new region we are able to obtain our result, $\alpha<1 / 9$. In $\$ 3$ we shall give a proof for the estimation of a certain integral over $E_{3}$ and in $\$ 4$ known integral estimations over the remaining regions, $E_{1}, E_{2}, E_{4}$, will be used.

\section{The integral over $E_{3}$}

LEMMA 1. If integers $b, r$ satisfy $(b, r)=1,1 \leq r$, and if $2 \leq Y$, then

$$
\sum_{p \leq y} e(b p / r) \log p \ll\left(r^{1 / 2} y^{1 / 2}+y^{5 / 7} p^{3 / 14}+y_{p}^{-1 / 2}\right)(\log Y)^{17} .
$$

Proof. This follows immediately from Theorem 16.1 in [6].

Put

$$
\theta_{0}=0, \quad \theta_{m}=m(2-9 A) / 2, \quad x_{m}=\delta X^{1-4 A+\theta} \text {, }
$$


460

Kee-Wai Lou and Ming-Chit Li u

$$
E_{3 m}=\left\{x:|x| \in\left(x_{m-1}, x_{m}\right]\right\} \text {, }
$$

where $m=1,2, \ldots, N=[2(5 A-1) /(2-9 A)]+1$. We see that

$$
1-4 A+\theta_{N}>A \text {. }
$$

So

$$
\bigcup_{m=1}^{N} E_{3 m} \supset E_{3} .
$$

LEMMA 2. If $x \in E_{3 m}$, then

$$
\min \left(\left|S_{1}(x)\right|,\left|S_{2}(x)\right|\right) \ll x^{1-A / 2+3 \theta} m^{/ 4} L^{17}
$$

Proof. Put

$$
Q=X^{l-A} \text {. }
$$

For each $x \in E_{3 m}$ and $j=1,2$, by Theorem 36 in [4] there are $\left(a_{j}, q_{j}\right)=1, I \leq a_{j} \leq Q$, such that

$$
\left|\lambda_{j} x-a_{j} q_{j}^{-1}\right| \leq q_{j}^{-1} Q^{-1}
$$

We see that

$$
a_{1} a_{2} \neq 0
$$

for if $a_{1}=0$, then by $(3.4),(3.3)$, and (2.2),

$$
|x| \leq\left|\lambda_{1}\right|^{-1} Q^{-1}<\delta X^{1-4 A} .
$$

This is impossible as $x \in E_{3 m}$.

Next suppose that

$$
\max \left(q_{1}, q_{2}\right) \leq X^{A-m(2-9 A) / 4} .
$$

Write

$$
\begin{aligned}
a_{2} q_{1}\left(\lambda_{1} / \lambda_{2}\right)-a_{1} q_{2}=\left(a_{2} / q_{2}\right)\left(q_{1} q_{2} / \lambda_{2} x\right)\left(\lambda_{1} x-\left(a_{1} / q_{1}\right)\right) & \\
& -\left(a_{1} / q_{1}\right)\left(q_{1} q_{2} / \lambda_{2} x\right)\left(\lambda_{2} x-\left(a_{2} / q_{2}\right)\right)=T_{1}-T_{2},
\end{aligned}
$$

https://doi.org/10.1017/S0004972700008984 Published online by Cambridge University Press 
say. By $(3.4),(3.6)$, and (3.3), we have

$$
\begin{aligned}
\left|T_{1}\right| \leq\left(\left|\lambda_{2} x\right|+q_{2}^{-1} Q^{-1}\right)\left(q_{1} q_{2} /\left|\lambda_{2} x\right|\right) q_{1}^{-1} Q^{-1}=\left(q_{2}+Q^{-1}\left|\lambda_{2} x\right|^{-1}\right) Q^{-1} & \\
& <x^{2 A-1-m(2-9 A) / 4} .
\end{aligned}
$$

Similarly we have $\left|T_{2}\right| \ll x^{2 A-1-m(2-9 A) / 4}$.

Hence, in view of (2.3), we have

$$
\left|a_{2} q_{1}\left(\lambda_{1} / \lambda_{2}\right)-a_{1} q_{2}\right| \ll x^{2 A-1-m(2-9 A) / 4}<1 / 2 q .
$$

Now for any integers $a^{\prime}, q^{\prime}$ satisfying $1 \leq q^{\prime}<q$, by (2.1) we have (3.8) $\left|q^{\prime}\left(\lambda_{1} / \lambda_{2}\right)-a^{\prime}\right| \geq q^{\prime}\left(\left(\left|a^{\prime} q-a q^{\prime}\right| / q q^{\prime}\right)-\left|(a / q)-\left(\lambda_{1} / \lambda_{2}\right)\right|\right)$

$$
>q^{\prime}\left(\left(1 / q q^{\prime}\right)-\left(1 / 2 q^{2}\right)\right)>1 / 2 q .
$$

Put $q^{\prime}=\left|a_{2} q_{1}\right|$ and $a^{\prime}= \pm a_{1} q_{2}$. By (3.5) we see that $1 \leq q^{\prime}$. It follows from $(3.7),(3.8)$, and (2.3) that

$$
\left|a_{2} q_{1}\right| \geq q=X^{1-2 A} \text {. }
$$

But by $(3.4),(3.6),(3.1)$, and $x \in E_{3 m}$, we have

(3.10) $\left|a_{2} q_{1}\right|=\left|\left(a_{2} / q_{2}\right)\right| q_{1} q_{2} \leq\left(\left|\lambda_{2} x\right|+Q^{-1} q_{2}^{-1}\right) q_{1} q_{2} \ll x_{m} X^{2 A-m(2-9 A) / 2}$

$$
<\delta X^{1-2 A}<q \text {. }
$$

Since (3.10) contradicts (3.9), (3.6) must be false. Therefore we may assume that

$$
x^{A-m(2-9 A) / 4}<q_{1}\left(\leq X^{1-A}\right)
$$

Put

$$
c_{n}= \begin{cases}e\left(a_{1} p / q_{1}\right) \log p & \text { if } n \text { is a prime } p \\ 0 & \text { otherwise, }\end{cases}
$$

and $z=\lambda_{1} x-\left(a_{1} / q_{1}\right)$. By Theorem 421 in [4], Lemma 1 (with $b=a_{1}$, $\left.r=q_{1}\right)$, and $(3.4)$, we have 


$$
\begin{aligned}
s\left(\lambda_{1} x\right) & =\sum_{n \leq X} c_{n} e(n z)=e(X z) \sum_{p \leq X} e\left(a_{1} p / q_{1}\right) \log p \\
& \quad-\int_{1}^{X} 2 \pi i z e(Y z)\left\{\sum_{p \leq Y} e\left(a_{1} p / q_{1}\right) \log p\right\} d Y \\
& <\left(q_{1}^{1 / 2} X^{1 / 2}+X^{5 / 7} q_{I}^{3 / 14}+X q_{1}^{-1 / 2}\right\} L^{17}\left\{1+X q_{1}^{-1} Q^{-1}\right\} .
\end{aligned}
$$

It follows from (3.11), (3.3), and (3.1) that

$$
\begin{aligned}
S\left(\lambda_{1} x\right) & \ll X^{1-A / 2+m(2-9 A) / 8_{L} 17_{X} m(2-9 A) / 4} \\
& \ll X^{1-A / 2+3 \theta^{/ 4}} L^{17} .
\end{aligned}
$$

This proves Lemma 2 .

LEMMA 3. We have

$$
\int_{x_{m-1}}^{x_{m}}|F(x)| K_{\varepsilon} d x \ll x^{2} \varepsilon^{2} L^{-1} .
$$

Then

$$
\int_{E_{3}}|F(x)| K_{\varepsilon} d x \ll X^{2} \varepsilon^{2} L^{-1}
$$

Proof. By (2.6), Parseval's identity, and $\sum_{p \leq X} 1 \ll X / L$, we have

$$
\int_{0}^{1}|S(y)|^{2} d y=\sum_{p \leq X}(\log p)^{2} \ll X L .
$$

So, by (2.5) and (3.1),

$$
\text { (3.14) } \begin{aligned}
\int_{x_{m-1}}^{x_{m}} & \left|S_{j}(x)\right|^{2} k_{\varepsilon} d x \\
& \ll \int_{y>\left|\lambda_{j}\right| x_{m-1}}^{\infty}|S(y)|^{2} y^{-2} d y \ll \sum_{n>\left|\lambda_{j}\right| x_{m-1}} n^{-2} \int_{n-1}^{n}|S(y)|^{2} d y \\
& \ll X L / x_{m-1} \ll x^{4 A-\theta_{m-1}} .
\end{aligned}
$$

On the other hand, note that 


$$
|F(x)| \ll \min \left(\left|S_{1}(x)\right|,\left|S_{2}(x)\right|\right) \sum_{j=1}^{3}\left|S_{j}(x)\right|^{2} .
$$

Then by Lemma 2, (3.14), and (3.1), we have

$$
\begin{aligned}
& \int_{x_{m-1}}^{x_{m}}|F(x)| K_{\varepsilon} d x \ll X^{1-A / 2+3 \theta_{m} / 4} L^{17}\left(X^{4 A-\theta_{m-1}} L\right) \\
& \ll X^{1+7 A / 2+(2-9 A)(4-m) / 8} L^{18} \ll X^{2} \varepsilon^{2} L^{-1} .
\end{aligned}
$$

The last inequality follows from (2.4) and

$$
1+7 A / 2+(2-9 A)(4-m) / 8<2-A .
$$

So (3.13) follows from (3.12) and (3.2).

\section{Completion of the proof}

LEMMA 4. If $x \in E_{2}$ then

$$
\min \left(\left|S_{1}(x)\right|,\left|S_{2}(x)\right|\right) \ll X^{1-A / 2} L^{17}
$$

Proof. The proof is similar to that of Lemma 2. But here we put $Q=\delta^{-1} X^{1-A}$ instead. Then following the same argument as that of Lemma 2 we must have

$$
\max \left(q_{1}, q_{2}\right)>X^{A}
$$

since $X=q^{1 /(1-2 A)}$. Then apply Lemma 1 .

LEMMA 5. We have

$$
\int_{E_{2}}|F(x)| K_{\varepsilon} d x \ll x^{2} \varepsilon^{2} L^{-1} .
$$

Proof. This follows from Lemma 4 and the same argument as that of Lemma 12 in [9].

LEMMA 6. We have

$$
\int_{E_{1}} e(x \eta) F(x) K_{\varepsilon} d x \gg x^{2} \varepsilon^{2} .
$$

Proof. Note that we may apply Lemmas 2 to 8 in [9] directly without 
any changes since these results do not depend on $E_{1}$.

Lemma 9 in [9] still holds if we replace $\tau$ there by our $\tau_{1}=X^{A-1}$. But we need to make a slight modification to (33) in [9] as follows. By Lemma 5 in [9] the integral in (33) is

$$
\begin{aligned}
& \ll X^{4 / 3} L^{C} \int_{-X^{A-1}}^{X^{A-1}}\left(1+X\left|\lambda_{j} x\right|\right)^{2} d x \\
& \ll X^{4 / 3} L^{C} X^{2} X^{3(A-1)}\left|\lambda_{j}\right|^{2} \\
& \ll x^{3 A+1 / 3} L^{C} \ll X L^{-2} .
\end{aligned}
$$

The last inequality follows from (2.2); that is, $A<2 / 9$. Then we continue the proof exactly as in [9, p. 379].

Finally, Lemma 10 in [9] still holds if we replace $\tau$ there by our $\tau_{1}$. No modification is necessary in the proof. Then Lerma 6 follows.

LEMMA 7. We have

$$
\int_{E_{4}}|F(x)| K_{\varepsilon} d x \ll x^{2} \varepsilon^{2} L^{-1} .
$$

Proof. This is Lemma 13 in [9].

LEMMA 8. For any real $y$ we have

$$
\int_{-\infty}^{\infty} e(x y) k_{\varepsilon} d x=\max (0, \varepsilon-|y|) \text {. }
$$

Proof. This is Lemma 1 in [10].

We come now to prove our theorem. By Lemma 8 and (2.7) we have

$$
\begin{aligned}
J & =\int_{-\infty}^{\infty} e(x \eta) F(x) K_{\varepsilon} d x \\
& =\sum_{\substack{p_{j} \leq X \\
j=1,2,3}}\left(\prod_{j=1}^{3} \log p_{j}\right) \max \left(0, \varepsilon-\left|\eta+\sum_{j=1}^{3} \lambda_{j} p_{j}\right|\right) \\
& \ll L^{3} \varepsilon N,
\end{aligned}
$$

where $N$ is the number of solutions in primes $p_{j}$ of 


$$
\left|\eta+\sum_{j=1}^{3} \lambda_{j} p_{j}\right|<\varepsilon \leq\left(\max p_{j}\right)^{-A / 2}\left(\max \log p_{j}\right)^{20}
$$

with $p_{j} \leq X \quad(j=1,2,3)$. So, by (2.2), that is $\alpha<A / 2$, our theorem follows if $J L^{-3} \varepsilon^{-1} \rightarrow \infty$ as $X \rightarrow \infty$. Now

$$
J=\sum_{\nu=1}^{4} \int_{E_{\nu}} e(x n) F(x) K_{\varepsilon} d x
$$

By Lemmas $5,3,7$, we have

$$
\sum_{\nu=2}^{4} \int_{E_{\nu}}|F(x)| K_{\varepsilon} d x \ll x^{2} \varepsilon^{2} L^{-1} .
$$

So Lemma 6, together with (4.1), shows that $J L^{-3} \varepsilon^{-1} \gg \chi^{2} \varepsilon L^{-3}$ as desired. This completes the proof of our theorem.

\section{Remark}

In $\S 3$ and in the proof of Lemma 6 we need $A<2 / 9$, which leads to our result $\alpha<1 / 9$ (see (2.2)). In fact, in the proof of Lemma 6 we can replace $A<2 / 9$ by a better one, namely $A<(\sqrt{21}-1) / 15=2 /(8.37 \ldots)$ if we modify the argument as in $[5,84]$. So it seems that the first difficulty encountered in any further improvement lies in $\$ 3$.

\section{References}

[1] A. Baker, "On some diophantine inequalities involving primes", $J$. reine congew. Math. 228 (1967), 166-181.

[2] R.J. Cook, "Diophantine inequalities with mixed powers", J. Number Theory 9 (1977), 142-152.

[3] H. Davenport and $H$. Heilbronn, "On indefinite quadratic forms in five variables", J. London Math. Soc. 21 (1946), 185-193.

[4] G.H. Hardy and E.M. Wright, An introduction to the theory of numbers, 4 th edition (Clarendon Press, Oxford, 1960).

[5] Ming-Chit Liu, "Approximation by a sum of polynomials involving primes", J. Hath. Soc. Japan 30 (1978), 395-412. 
[6] Hugh L. Montgomery, Topics in multiplicative number theory (Lecture Notes in Mathematics, 227. Springer-Verlag, Berlin, Heidelberg, New York, 1971).

[7] K. Ramachandra, "On the sums $\sum_{j=1}^{K} \lambda_{j} f_{j}\left(p_{j}\right)$ ",J. reine angew. Math. $262 / 263(1973), 158-165$.

[8] Wolfgang Schwarz, "Über die Lösbarkeit gewisser Ungleichungen durch Primzahlen", J. reine angew. Math. 212 (1963), 150-157.

[9] R.C. Vaughan, "Diophantine approximation by prime numbers, I", Proc. London Math. Soc. (3) 28 (1974), 373-384.

[10] R.C. Vaughan, "Diophantine approximation by prime numbers, II", Proc. London Math. Soc. (3) 28 (1974), 385-401.

[11] R.C. Vaughan, "Diophantine approximation by prime numbers, III", Proc. London Math. Soc. (3) 33 (1976), 177-192.

Department of Mathematics,

University of Hong Kong,

Hong Kong. 